\title{
Fluorine Substituted Proline Enhances Tubulin Binding Potential of a Tetrapeptide at GTP Binding Pocket Causing Inhibition of Microtubule Motility and Antimitotic Effect
}

Batakrishna Jana1, ${ }^{1, \#}$ Surajit Barman, ${ }^{1, \#}$ Rajsekhar Roy, ${ }^{2}$ Gaurav Das, ${ }^{1,3}$ Nabanita Mukherjee, ${ }^{2}$

Anindyasundar Adak, ${ }^{1}$ and Surajit Ghosh, 2, $3 *$

${ }^{1}$ Organic and Medicinal Chemistry and Structural Biology and Bioinformatics Division, CSIRIndian Institute of Chemical Biology, 4, Raja S. C. Mullick Road, Jadavpur, Kolkata-700 032, WB, India. Fax: +91-33-2473-5197/0284; Tel: +91-33-2499-5872

${ }^{2}$ Department of Bioscience \& Bioengineering, Indian Institute of Technology Jodhpur, NH 62, Surpura Bypass Road, Karwar, Rajasthan 342037, India, Phone: +91-291-280-1212

${ }^{3}$ Academy of Scientific and Innovative Research (AcSIR), Ghaziabad 201002, India

${ }^{\$}$ Present address (B.J.): Department of Chemistry, UNIST, Ulsan 44919, Republic of Korea

\#These authors contributed equally.

CORRESPONDING AUTHOR INFORMATION:

E-mail: sghosh@iitj.ac.in 
Chemicals: (2S, 4S) - Fmoc-4-trifluoromethyl-pyrrolidine-2-carboxylic acid was purchased from PolyPeptide group. $\mathrm{NaCl}, \mathrm{NaHCO}_{3}, \mathrm{NaOH}, \mathrm{Na}_{2} \mathrm{HPO}_{4}, \mathrm{KOH}, \mathrm{C}_{6} \mathrm{H}_{5} \mathrm{OH}, \mathrm{EDT}$, TFA, $\mathrm{C}_{2} \mathrm{H}_{5} \mathrm{OH},\left(\mathrm{CH}_{3}\right)_{2} \mathrm{CO}$, DCM, DMF, $\mathrm{MgCl}_{2}$ and $\mathrm{KH}_{2} \mathrm{PO}_{4}$ were purchased from Merck. $\mathrm{KCl}$ and Ether were purchased from Fisher Scientific. Pipyridine, DMSO, HBTU and DIPEA were purchased from Spectrochem. $\mathrm{CH}_{3} \mathrm{CN}$ was purchased from J. T. Baker. Triton-X-100 was purchased from SRL. (2S, 4S)-Fmoc-4-fluoro-pyrrolidine-2-carboxylic acid, Fmoc-D-Pro-OH, 3-(4, 5-dimethylthiazol-2-yl)-2, 5-diphenyltetrazolium bromide (MTT), Kanamycin Sulfate, Dulbecco's Modified Eagle's Medium (DMEM) medium, Trypsin-EDTA solution, ATP, GTP, PIPES, EGTA, cell cultured DMSO, DAPI and formaldehyde were purchased from MerckSigma Aldrich. 2-[4-(2-hydroxyethyl) piperazin-1-yl] ethanesulfonic acid (HEPES) was purchased from Himedia. Bisbenzimide H 33258 (Hoechst) was purchased from Calbiochem. p53 (F-8) mouse monoclonal IgG, p21 (F-5) mouse monoclonal IgG, goat anti mouse IgG $(\mathrm{H}+\mathrm{L}) \mathrm{RPE}$, human adsorbed antibody and annexin $\mathrm{V}$ apoptosis detection kit were purchased from Santa Cruz Biotechnology. All compounds were used without further purification. 


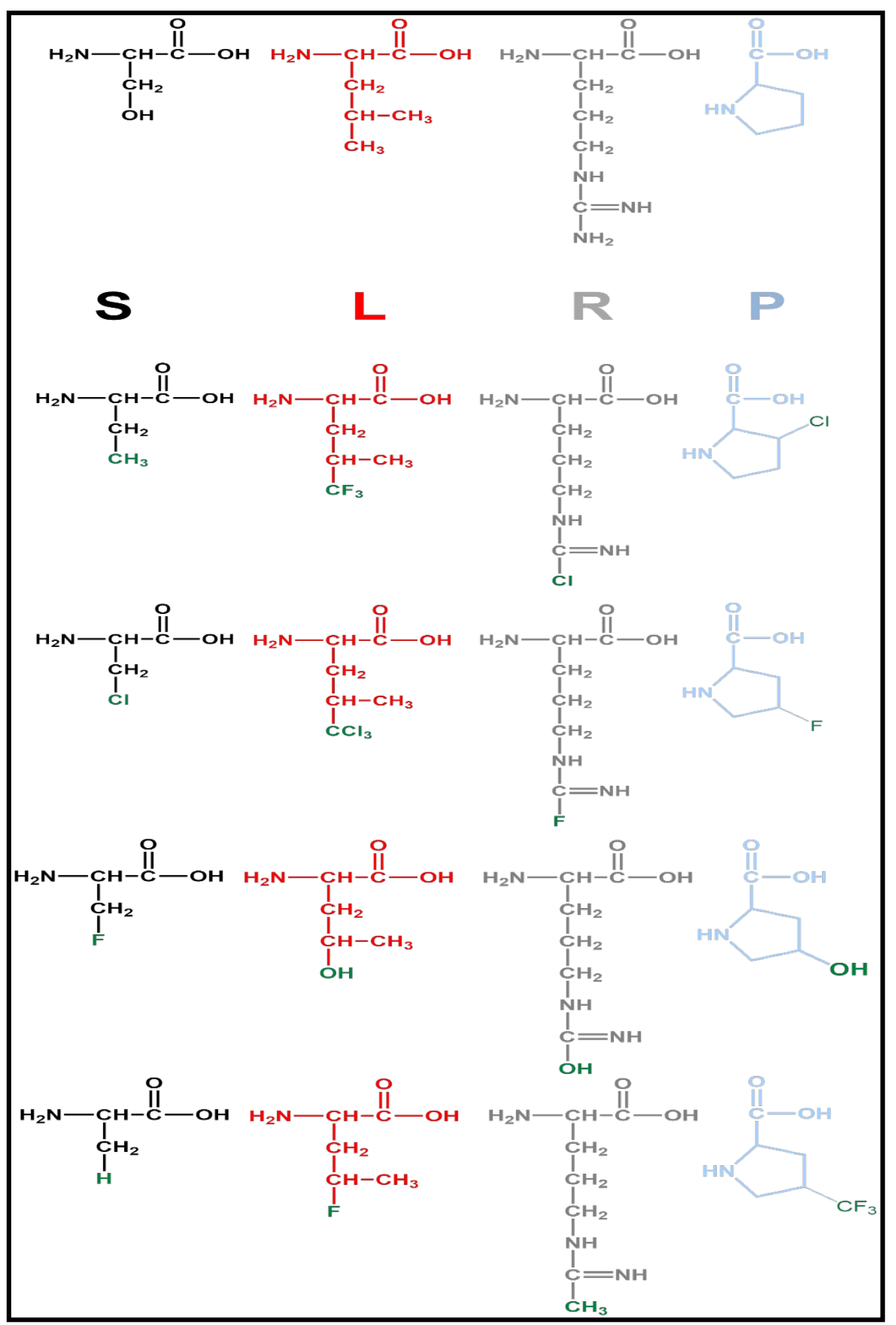

Figure S1. Modified clusters that were used to perform de novo design to determine the components of the tetra-peptide. This ultimately showed the components for $\mathrm{SP}_{-} \mathrm{CF}_{3}$ as the favorable one. 
Peptide analogues

$$
\begin{gathered}
\text { SP } \\
\text { SP-D } \\
\text { SP-F } \\
\text { SP-OH } \\
\text { SP-CF }_{3}
\end{gathered}
$$

binding energy with tubulin

$$
(\mathrm{Kcal} / \mathrm{mole})
$$

$-7.8$

Figure S2. The table showing the various binding energy of the peptide analogues of SP with the tubulin. The result showing that $\mathrm{SP}_{-} \mathrm{CF}_{3}$ has the lowest binding energy (highest binding affinity) with tubulin among the peptide analogues.

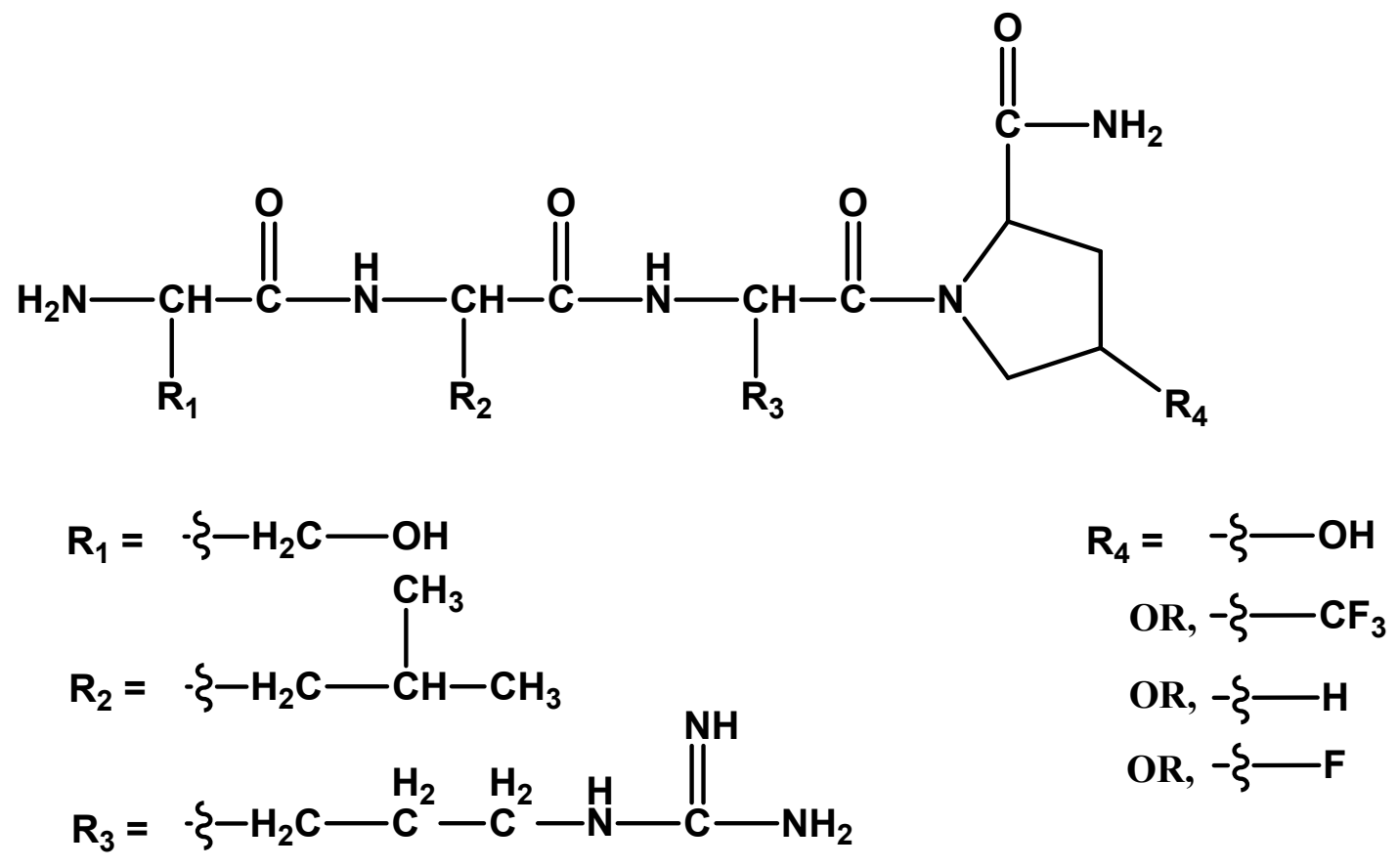

Figure S3. Structure of SP-OH, SP-CF 3 , SP-D and SP-F. 


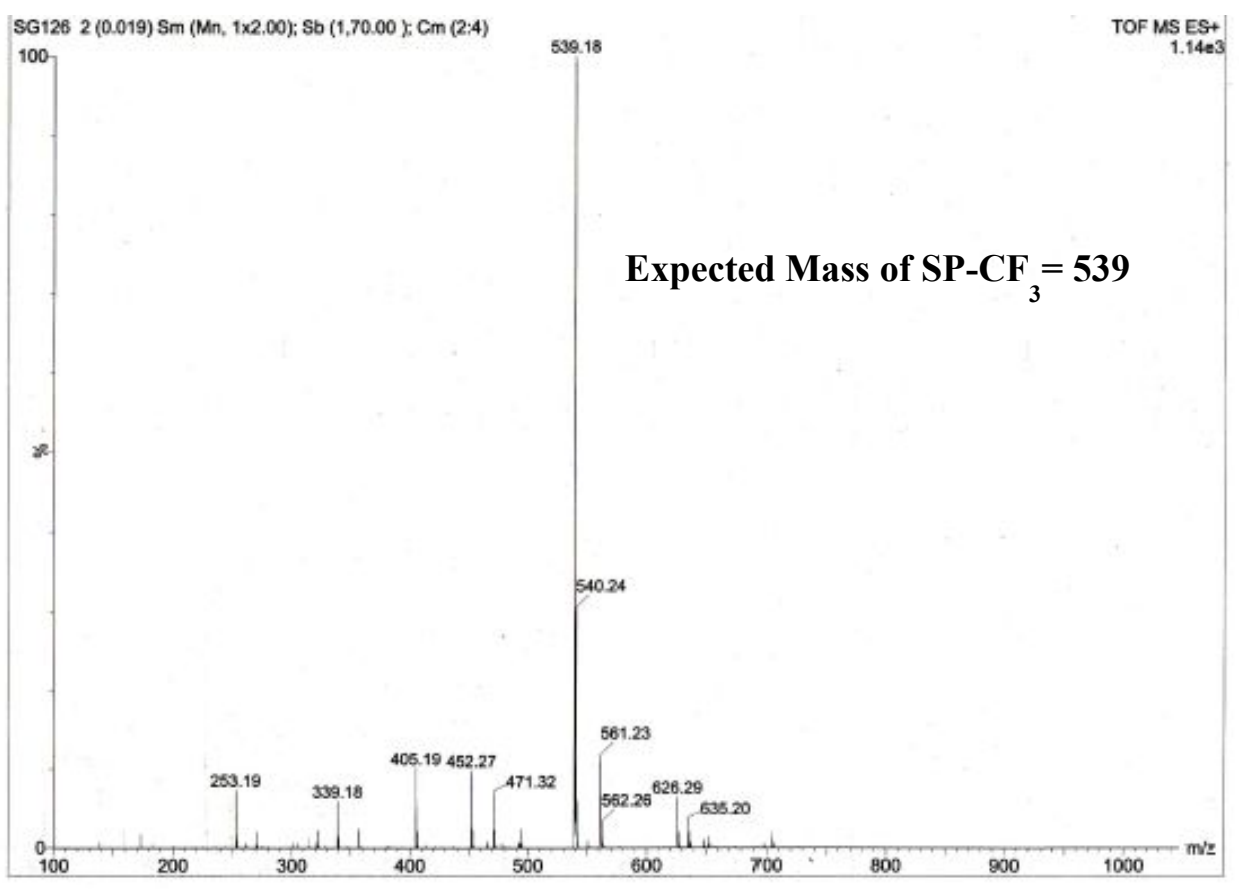

Figure S4. ESI mass spectrum of $\mathrm{SP}-\mathrm{CF}_{3}$.

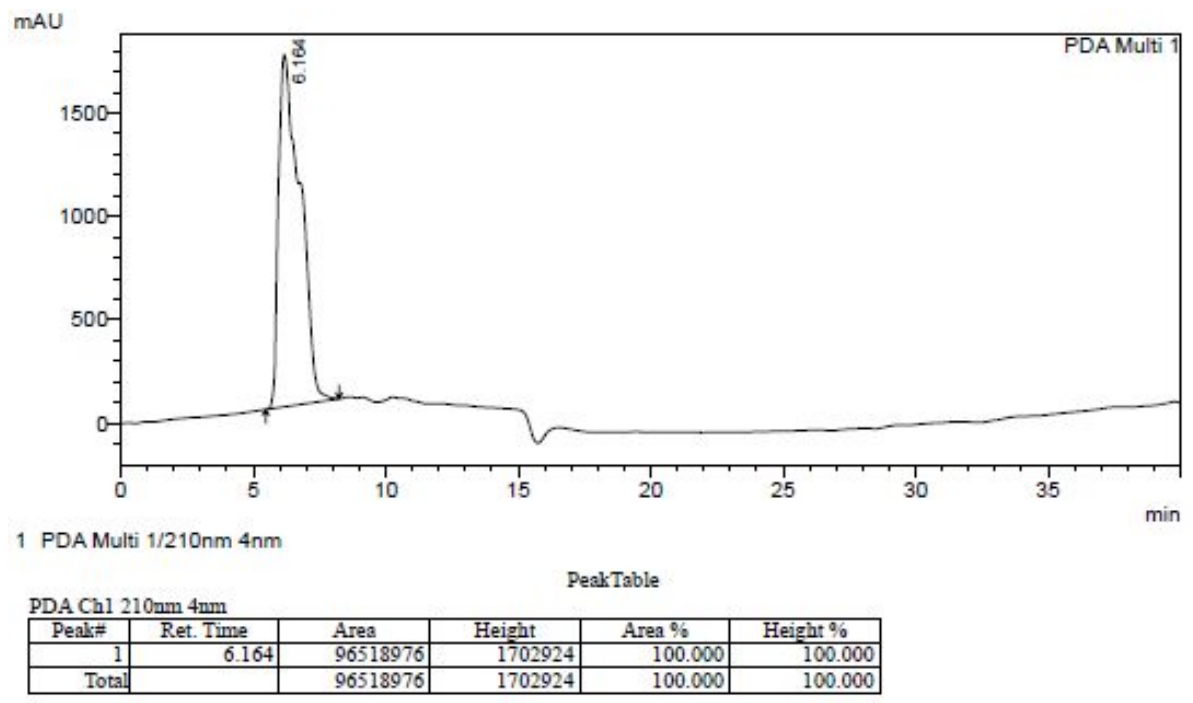

Figure S5. HPLC chromatogram of SP-CF 3 . 


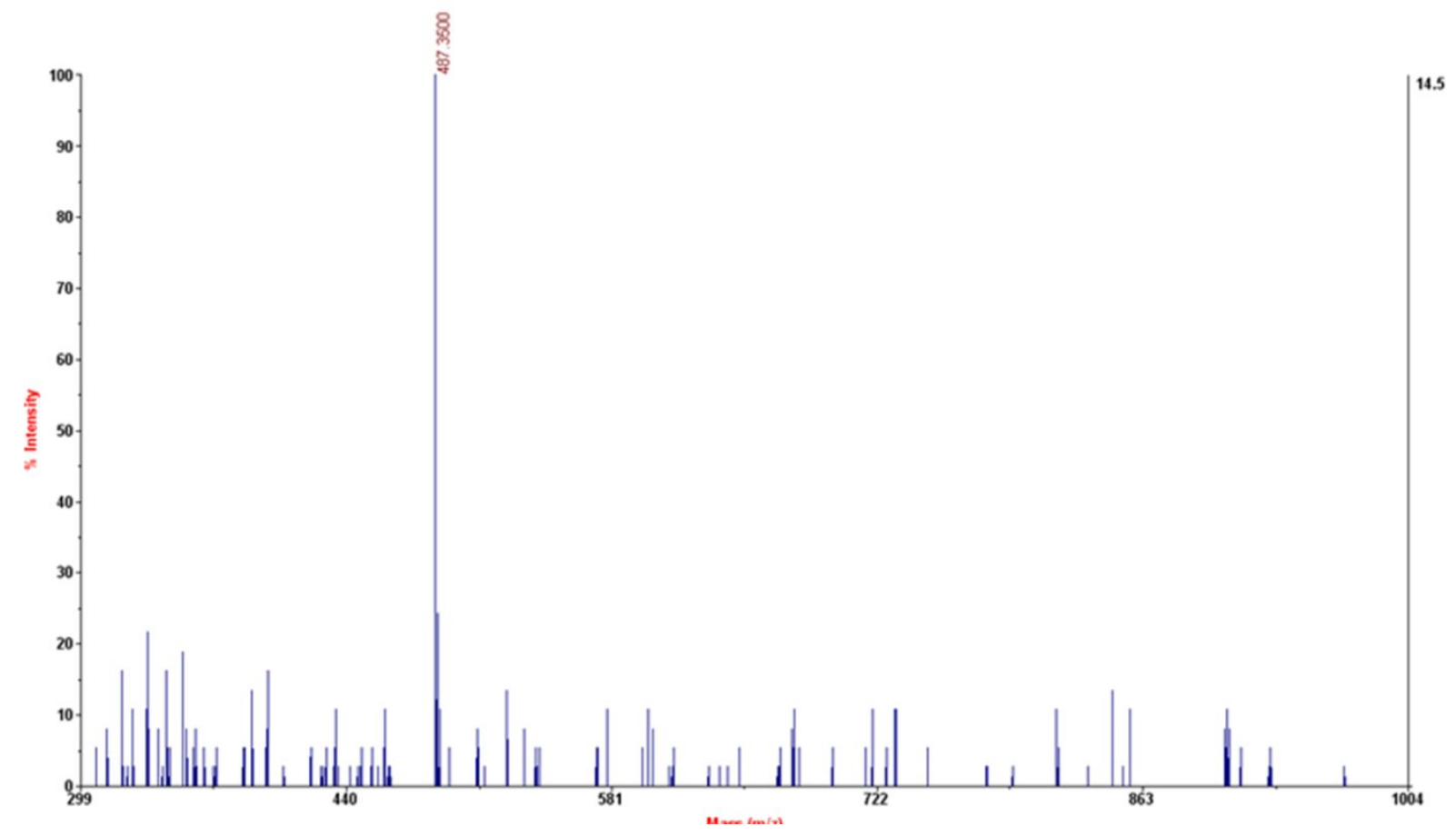

Figure S6. MALDI mass spectrum of SP-OH.

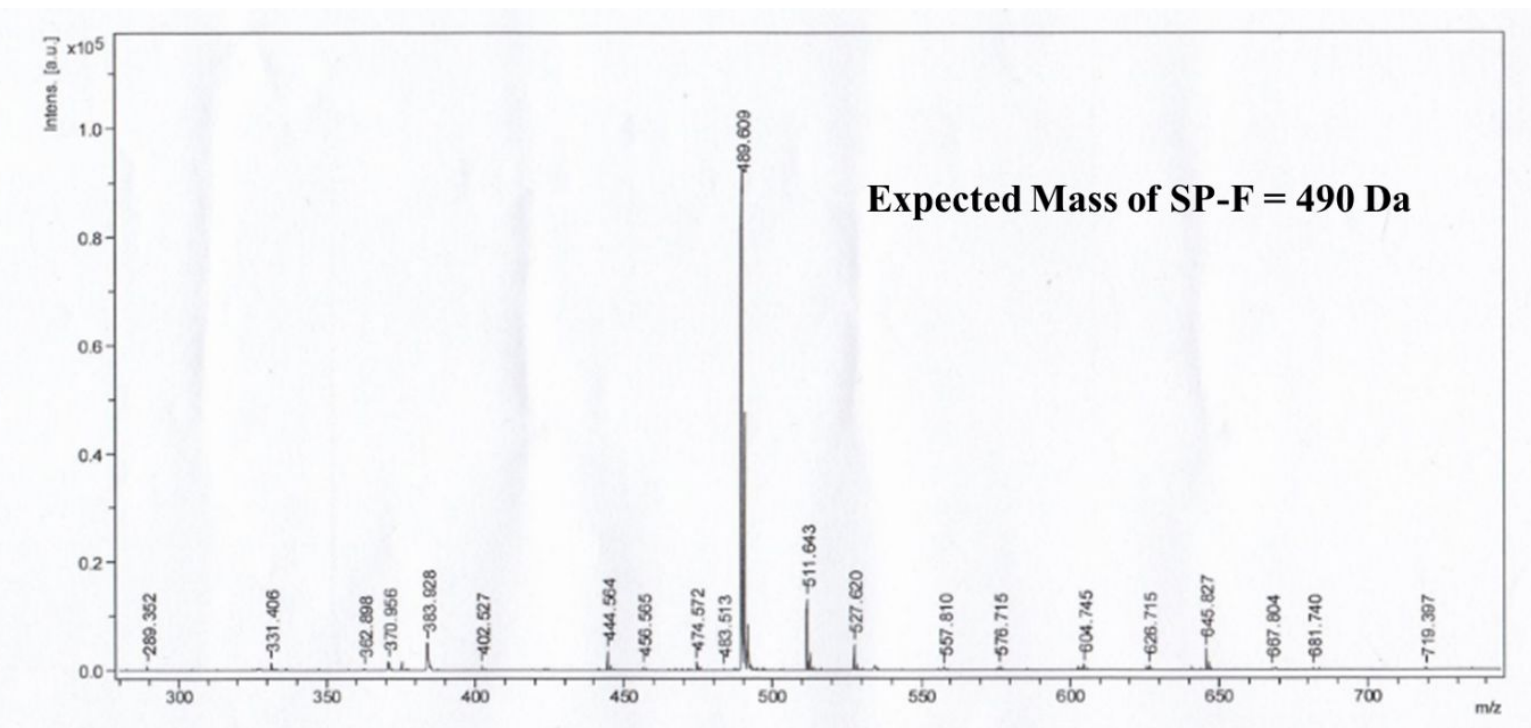

Figure S7. MALDI mass spectrum of SP-F. 


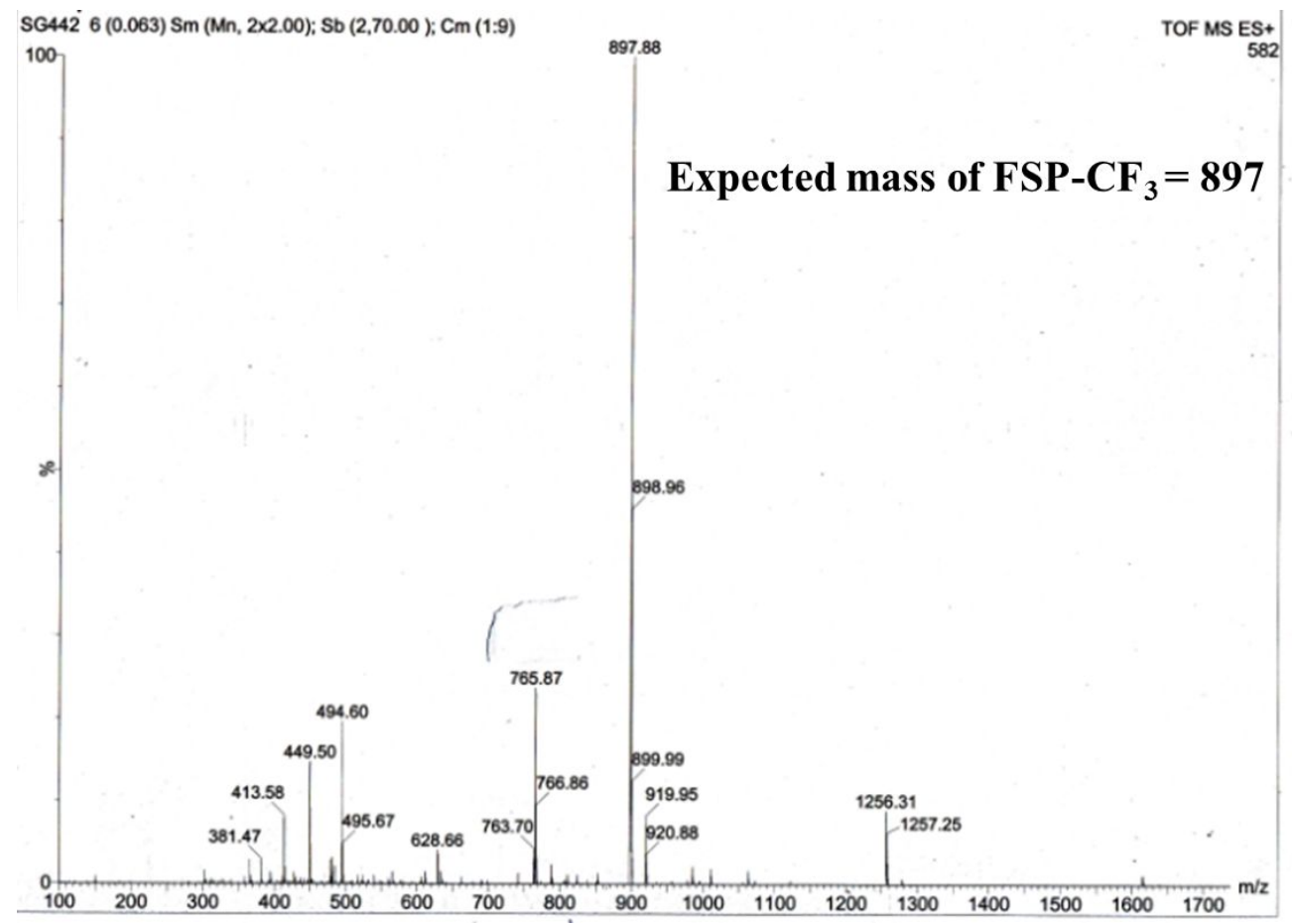

Figure S8. ESI mass spectrum of FSP-CF ${ }_{3}$.

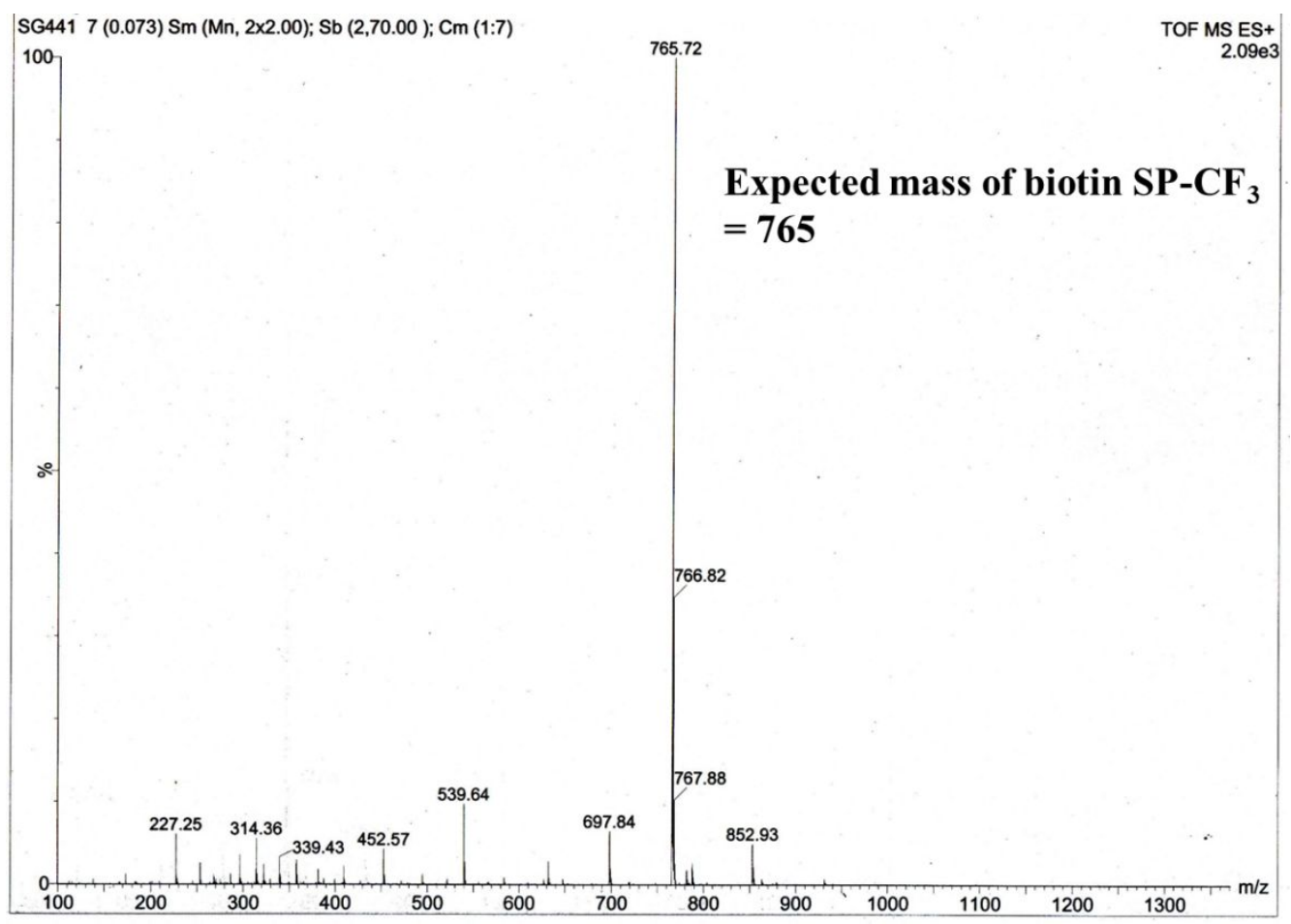

Figure S9. ESI mass spectrum of biotin $\mathrm{SP}-\mathrm{CF}_{3}$. 


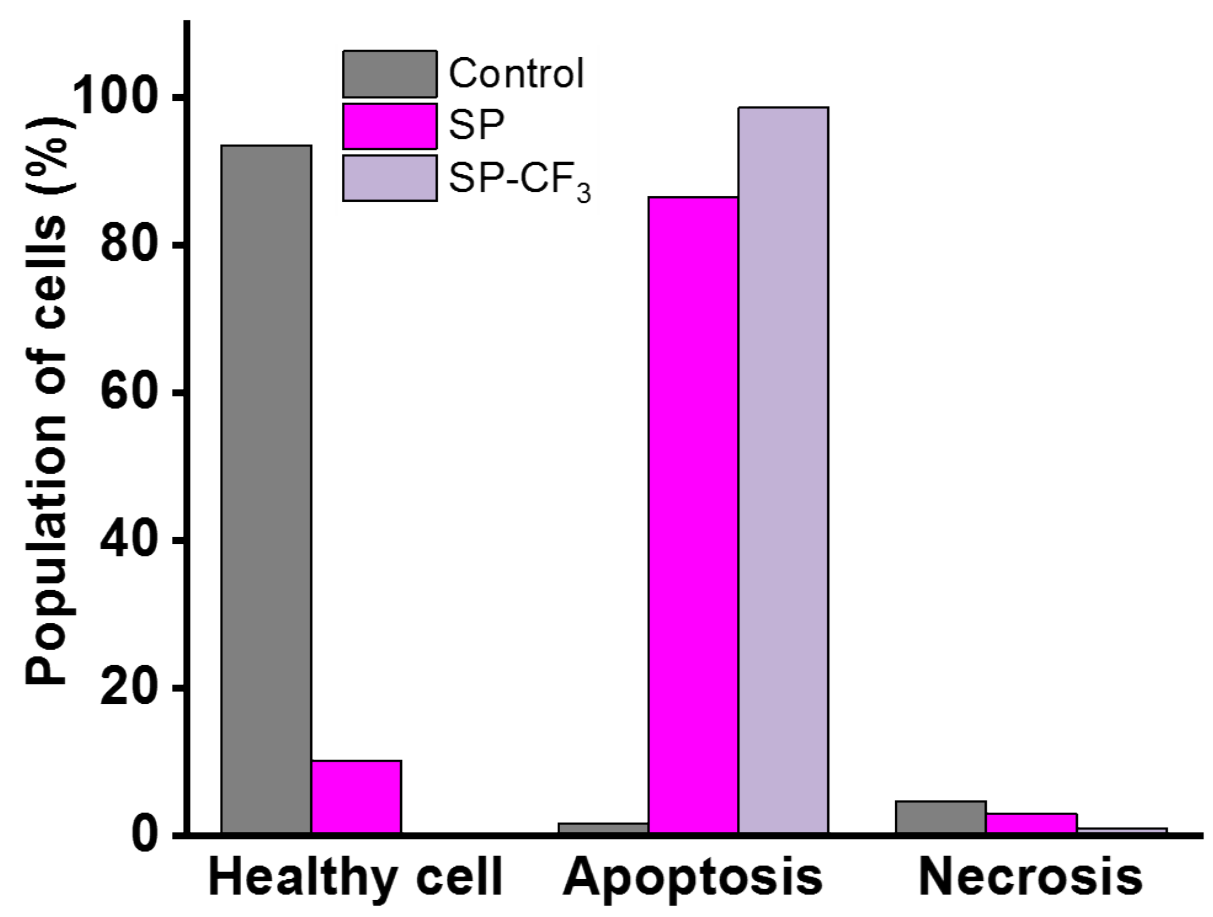

Figure S10. Histogram of percentages of healthy, apoptotic and necrotic cells after SP and SP$\mathrm{CF}_{3}$ treatment in MCF-7 cells, measured using flow cytometry. 